\title{
Assessing the Role of Conceptual Knowledge in an Anti-Phishing Educational Game
}

\author{
Michael James Scott, Gheorghita Ghinea \\ Department of Computer Science \\ Brunel University \\ London, United Kingdom \\ \{michael.scott, george.ghinea\}@brunel.ac.uk
}

\author{
Nalin Asanka Gamagedara Arachchilage \\ Cyber Security Centre \\ Oxford University \\ Oxford, United Kingdom \\ nalin.gamagedaraarachchilage@cs.ox.ac.uk
}

\begin{abstract}
Games can be used to support learning in several domains, including the secure use of computers. However, emphasizing different types of knowledge in a game design can lead to different outcomes. This study explores two game designs that aim to enhance students' ability to identify phishing hyperlinks. One design focuses on procedural knowledge: developing students' tacit ability to recognize phishing hyperlinks through systematic practice. The other design focuses on conceptual knowledge: helping students to explicitly reflect upon and identify the features of phishing hyperlinks. The results of a double-blind randomized trial with 66 participants suggests that using a game designed for conceptual knowledge leads to a greater increase in learners' ability to identify phishing hyperlinks. Hence, the use of strategies that develop conceptual knowledge can enhance the efficacy of anti-phishing educational games.
\end{abstract}

Keywords-procedural knowledge; conceptual knowledge; game-based learning; phishing; education; games.

\section{INTRODUCTION}

Phishing is a type of identity theft that affects websites, in which unsuspecting individuals are deceived into sending confidential and sensitive information to a third party website as it fraudulently pretends to be another. Typically, an attack presents an individual with a hyperlink which appears to be associated with a website they are familiar with, but is actually associated with another.

Game-based learning tools can be used to help students thwart such phishing attacks [1]. However, different design choices made in these games could emphasize different types of knowledge and lead to different outcomes [2]. Those designs placing emphasis on experiential learning through a systematic program of practice activities may help students to develop procedural knowledge; a type of knowledge that is tacit (rather than verbalized) but enables students to recognize actions and rules as they complete a task [2]. Those designs placing emphasis on information, complex problem solving and reflection help students develop their conceptual knowledge; a type of knowledge which can be verbalized (rather than being tacit) and facilitates the formation of rich connections within a domain [2].

An existing anti-phishing educational game explicitly focuses on conceptual knowledge [1], despite the framework used to inform its design [3] providing no sound justification for this choice. As such, it is not clear which emphasis is more appropriate within this computer security context.

\section{FINDINGS}

To investigate, a pre-post test double-blind randomised trial, incorporating balanced allocation between two parallel groups, was conducted to examine the impact of two games: a design incorporating features that emphasize conceptual knowledge (experimental condition) and an ablated version of the same game omitting such features (control condition).

A random sample of 66 first-year undergraduate students (age 18+) at Brunel University were recruited via email. There was a response rate of $26.4 \%$.

The data was analyzed using PASW 18.0.3 for Windows. All cases were included (no missing data). Table I shows the results of an ANCOVA examining differences in test scores (identifying 15 phishing hyperlinks) between the groups:

TABLE I. $\quad$ ANCOVA RESULTS $(\mathrm{DV}=$ POST-TEST $)$

\begin{tabular}{lrrrrr}
\hline \hline Source & $S S$ & $M S$ & $F$ & $p$ & $\eta_{\mathrm{p}}{ }^{2}$ \\
\hline Pre-Test & 322.90 & 322.90 & 155.470 & .000 & .712 \\
Experimental Allocation & 20.43 & 20.43 & 9.838 & .003 & .135 \\
Error & 130.84 & & & & \\
\hline Note: SS: Sum of Squares; MS: Mean Square. Adjusted Model R ${ }^{2}=.719$ &
\end{tabular}

These results show a statistically significant difference, in favor of the experimental group $(\Delta \mu=-1.114, p=.003)$, explaining approximately $13.5 \%$ of the variance.

\section{CONCLUSION}

This study indicates that an anti-phishing educational game incorporating design elements that privilege conceptual knowledge over procedural knowledge could better prepare students to identify phishing hyperlinks. This suggests that the development of conceptual knowledge may have an important role in educational games, at least within the computing security context.

\section{REFERENCES}

[1] N.A.G. Arachchilage, S. Love and M.J. Scott, "Designing a Mobile Game to Teach Conceptual Knowledge of Avoiding Phishing Attacks", International Journal for e-Learning Security, vol. 2, no. 1, June 2012, pp. 127-132.

[2] T. de Jong and M.G.M. Ferguson-Hessler, "Types and Qualities of Knowledge", Educational Psychologist, vol. 31, no. 2, May 1996, pp. 105-113.

[3] N.A.G. Arachchilage and S. Love, "A game design framework for avoiding phishing attacks", Computers in Human Behavior, vol. 29, no. 3, May 2013, pp. 706-714. 